\title{
Treatment Costs for Patients with Chronic Kidney Disease Who Received Multidisciplinary Care in a District Hospital in Thailand
}

This article was published in the following Dove Press journal: ClinicoEconomics and Outcomes Research

\section{Suwaporn Songsermlosakul (D) Unchalee Permsuwan (D) Wanchana Singhan}

Faculty of Pharmacy, Chiang Mai University, Chiang Mai, 50200, Thailand
Correspondence: Wanchana Singhan Email swnaice@gmail.com
Aim: To estimate direct medical treatment costs in patients with pre-dialysis chronic kidney disease (CKD) in a district hospital and to analyze the factors that affected the treatment costs.

Patients and Methods: Data were retrospectively retrieved from the hospital database in the period from January 2015 to December 2017. Patients who were diagnosed with CKD and had visited ambulatory care services at least two times during the index year (January to December 2015) were included. Patients' data were excluded if they had cancer, had received renal replacement therapy, or had been referred to receive treatment at other hospitals. Treatment costs based on the providers' perspectives in the first and second years after the index year were assessed. Descriptive statistics were used to analyze patients' characteristics, and multiple linear regression was used to analyze the factors in the cost model.

Results: Data of 212 patients with CKD stage G3a, G3b, or G4 who met inclusion and exclusion criteria were included for analysis. Average costs for treatment in year 1 and year 2 were not statistically different. Total cost was 5701.34 Thai Baht (THB) per year. The total cost for patients with CKD stage G4 was two times greater than for patients with CKD stage G3. Costs were increased for longer hospitalization, more frequent ambulatory visits, having diabetes mellitus or dyslipidemia as a comorbidity, and uncontrolled fasting blood glucose (FBG). A cost model with $R^{2}=0.906$ was provided. Significant predictors were length of stay, ambulatory visits, diabetes mellitus, dyslipidemia, serum creatinine, FBG, and body mass index.

Conclusion: Total annual treatment costs for the 2 years were not different. A more advanced stage of CKD, having diabetes mellitus or dyslipidemia as comorbidities, and uncontrolled FBG were significantly associated with increased costs for treatment in patients with pre-dialysis CKD.

Keywords: cost, chronic kidney disease, district hospital, multidisciplinary

\section{Introduction}

Chronic kidney disease (CKD) is a chronic disease that causes a public health burden worldwide. Global CKD prevalence is approximately $13.4 \%{ }^{1}$ while it has increased to $17.7 \%$ in Thailand. ${ }^{2}$ Hypertension and diabetes mellitus are common comorbidities in CKD patients. Uncontrolled comorbidities precipitate CKD progression ${ }^{3,4}$ and lead to costly medical treatment, ${ }^{5-7}$ especially in dialysis patients. ${ }^{8-10}$

The multidisciplinary care (MDC) approach, compared with the usual care model, has shown benefits in terms of lower risk of all-cause mortality, lower rate of hospitalization, slower rate of glomerular filtration rate (GFR) decline, and less requirement for long-term dialysis. ${ }^{6,7,11,12}$ However, its benefit in cost savings 
is still controversial among different countries. The studies conducted in Taiwan indicated that the MDC approach has lower direct medical treatment costs for patients with advanced stages of CKD than the costs associated with usual care. ${ }^{6,11}$

A study conducted in the USA showed that the MDC approach was more cost-effective for patients with CKD stage G3 to G4 compared with usual care. ${ }^{7}$ Conversely, studies conducted in Germany reported greater direct medical costs incurred with MDC than those with usual care. ${ }^{13}$

Based on the scoping review of 40 studies in $2019,{ }^{14}$ there is inconsistency due to various MDC team compositions. In Thailand, the MDC approach has been utilized for a decade. Thai MDC teams are composed of 2 general practitioners, 2 chronic care nurses, 1 pharmacist, 1 physical therapist, and 1 nutritionist. ${ }^{15}$ Teams are aimed at reducing the incidence of $\mathrm{CKD}$ and end-stage renal disease, improving patients' quality of life, and increasing access to renal replacement therapy (RRT). From a previous randomized controlled trial in Thailand comparing integrated care with an MDC approach and usual care, ${ }^{16}$ the result shows that patients who received integrated care with MDC have better GFR over the 2 years of follow up. However, studies related to costs and outcomes of the MDC approach are still limited; therefore, this study aimed to estimate the direct medical treatment costs for patients with pre-dialysis CKD in Thailand.

\section{Patients and Methods}

\section{Study Design and Patients}

This retrospective cohort study was conducted at a public district hospital in Kamphaengphet province, Thailand. MDC members of CKD clinic in this setting comprised of 1 general practitioner, 2 nurses, 1 pharmacist, 1 dietician, and 1 physical therapist. There was also a home visit team that provide health care at patients' homes for every 3 months. Home visit team members comprised of 2 nurses or public health officers and at least 3 village health volunteers. Patients included in the study were aged 18 years or above, had been diagnosed with CKD (ICD 10 N138, N184 and N185) according to eGFR calculated by CKD-EPI equation, regularly received treatment at the aforementioned hospital, and had visited ambulatory care services at least two times during the index year (January to December 2015). Serum creatinine or GFR data were required to confirmed CKD stage. Patients were excluded if they met at least one of the following criteria: 1) were diagnosed with having cancer, 2) received RRT including chronic intermittent hemodialysis or peritoneal dialysis, 3) had been referred to other hospitals, or 4) were lost to follow up or death. CKD stages were classified by the recommendation of Kidney Disease Improving Global Outcomes (KDIGO). ${ }^{17}$ GFR was calculated using the CKD-EPI equation. ${ }^{18}$ The study protocol was in accordance with the Declaration of Helsinki and approved by the Institutional Review Board of Faculty of Pharmacy, Chiang Mai University (No. 010/2561). Since the hospital database was retrospectively retrieved in this study, written informed consent was not obtained from an individual patient. Code numbers were used in data collection to prevent individual identification and maintain patients' confidentiality.

\section{Data Collection}

Data were retrieved from the hospital database for 3 years starting from January 2015 to December 2017. The first year would be the index year. Patients who had visited the hospital in the index year and met the inclusion and exclusion criteria above would be included in this study. Demographic and clinical data of those included patients were collected in the first year; then costs would be collected for the next 2 years. Resource uses of these following items for both ambulatory care and in-patient care such as medications, parenteral nutrition, medical supplies, blood products, laboratory tests, and other medical services were collected. Unit costs of the items above were obtained from the standard unit cost list for medical and health services in Thailand. ${ }^{19}$

\section{Cost Estimation}

This study considered the health-care provider's perspective. Therefore, only direct medical costs were included. Direct medical costs comprised of cost in diagnosis, laboratory test, pharmacological and nonpharmacological treatment, and services for ambulatory care in outpatient clinics and emergency room, and hospitalization care. The cost estimation was analyzed by the multiplication of unit cost times the number of uses. Total cost was the summation of all cost items. Ambulatory cost was calculated for all patients, but hospitalization cost was calculated only for admitted patients. Costs were inflated using Thailand's consumer price index ${ }^{20}$ and presented in the year 2019.

\section{Statistical Analysis}

Patients' characteristics and clinical data were reported as descriptive statistics. Annual costs of different years and patients' characteristics were analyzed using the 
Independent $t$-test or the analysis of variance test as appropriate. Multiple linear regression analysis was used for forecasting cost models in CKD patient care. Stepwise method was used to plugged variables in the model. Statistical analyses were executed using SPSS 17.0 (SPSS, Chicago, IL, USA) and statistical significance was considered if $P<0.05$.

\section{Results}

A total of 331 patients were eligible based on inclusion criteria. However, 116 patients were excluded due to the following reasons: 1) referral to other settings (53 patients), 2) having cancer (52 patients), 3) receiving RRT (11 patients), and 4) loss to follow up or death (3 patients). Therefore, 212 patients with CKD stage G3 to G4 were included in the analysis. The average age was 69.42 years and $35.40 \%$ were male. Hypertension (93.90\%) was the most common comorbidity found in this group of patients. Diabetes mellitus was doubled in patients with CKD stage G4 compared with patients with CKD stage G3. Of the 41 total admissions, patients with stage G3a, G3b, and G4 were accounted for at 20 (48.78\%), $12(29.27 \%)$, and $9(21.95 \%)$, respectively. Patients' characteristics are shown in Table 1.

Table 2 shows that the average total treatment costs in year 1 and year 2 were 5701.34 and 5697.24 THB per year, respectively. Total costs were divided into ambulatory and hospitalization costs. It was found that ambulatory costs were greater than hospitalization costs for both years. However, total costs in year 1 and year 2 were not statistically significantly different $(P>0.05)$.

Ambulatory costs for patients aged less than 65 years were higher than those of patients aged more than 65 years (4902.94 and 3995.19 THB per year, $P=0.018$ ). Ambulatory costs, hospitalization costs, and total costs for treatment were higher for patients who had been hospitalized, especially those who had lengths of stay for more than 8 days, as opposed to those who had not been hospitalized. Costs were also higher for patients who had visited ambulatory services more than eight times per year, had diabetes mellitus as a comorbidity, and/or uncontrolled fasting blood glucose (FBG) which is defined as FBG > $130 \mathrm{mg} / \mathrm{dL}$. Treatment costs by patients' characteristics are shown in Table 3 and Supplementary Table 1.

Although 15 variables of patients' characteristics and clinical data obtained from the hospital database were plugged into the cost model, 6 variables demonstrated significant predictors at $P$-value $<0.05$. Those were age, length of stay, ambulatory visits, body mass index, diabetes mellitus, and FBG (Table 4 and Supplementary Table 2).

\section{Discussion}

The study estimated the direct medical costs for standard treatment with the MDC approach in patients with CKD stage G3 to G4 who received treatment at a district hospital. In Thailand, patients with an advanced CKD stage or who need RRT are referred to receive treatment with nephrologists at tertiary hospitals; hence, only CKD stages G3 and G4 patients receive treatment at district hospitals.

The average age of our sample population was 69.42 years, which was older than those in other studies. ${ }^{6,11,16}$ We found that all samples remained in the same CKD stage and received the same standard treatment over the 2 years. This led to no statistically significant difference in the estimated treatment costs.

More severe stages of CKD resulted in higher treatment costs. Although treatment costs for patients with CKD stage G3a and G3b were not different, it was doubled in patients with CKD stage G4 due to a higher hospitalization rate $(16.26 \%, 17.91 \%$, and $40.92 \%$ for patients with stage G3a, G3b, and G4, respectively). This finding was concordant with previous studies that estimated direct medical costs for treatment with the MDC approach. $^{7,13}$

The cost model showed that ambulatory visits, hospitalization, diabetes mellitus, dyslipidemia, serum creatinine, FBG, and body mass index are the main cost drivers. The more frequent ambulatory visits, the longer the hospitalization, having diabetes mellitus or dyslipidemia as a comorbidity, having higher serum creatinine or FBG, and greater body mass index independently increase treatment costs.

One-third of these CKD patients had diabetes mellitus and treatment costs were doubled compared to those with no diabetes mellitus. This might be due to a longer duration of hospitalization (1.20 vs 0.46 days) and more frequent ambulatory visits (6.81 vs 6.22 visits). Unexpectedly, patients with both CKD and diabetes mellitus who achieved the hemoglobin A1c (HbA1c) target had no significant difference in treatment costs compared with those who did not achieve the HbAlc target. This might be attributable to a small sample size (Table 1) and criteria. Due to FBG monitoring being more convenient and less expensive than HbA1c monitoring, it can be measured 
Table I Patients' Characteristics

\begin{tabular}{|c|c|c|c|c|c|}
\hline Characteristics & CKD G3a $(n=123)$ & CKD G3b $(n=67)$ & CKD G4 (n=22) & All $(n=2 \mid 2)$ & $P$-value \\
\hline Male sex (n) & $50(40.70)$ & $17(25.40)$ & $8(36.40)$ & $75(35.40)$ & 0.110 \\
\hline Age (years) & $68.11 \pm 10.05$ & $72.00 \pm 9.70$ & $68.82 \pm 10.52$ & $69.42 \pm 10.10$ & $0.038^{*}$ \\
\hline$<65$ & $44(35.80)$ & $15(22.40)$ & $8(36.40)$ & $67(31.60)$ & \\
\hline$\geq 65$ & $79(64.20)$ & $52(77.60)$ & $14(63.60)$ & $145(68.40)$ & \\
\hline Hypertension (n) & $117(95.10)$ & $64(95.50)$ & $18(81.80)$ & $199(93.90)$ & $0.045^{*}$ \\
\hline Diabetes mellitus ( $n$ ) & $37(30.10)$ & $23(34.30)$ & $14(63.60)$ & $74(34.90)$ & $0.009 *$ \\
\hline Dyslipidemia (n) & $61(49.60)$ & $31(46.30)$ & $13(59.10)$ & $105(49.50)$ & 0.584 \\
\hline $\mathrm{MI} / \mathrm{CHF}(\mathrm{n})$ & I $(0.80)$ & $3(4.50)$ & $2(9.10)$ & $6(2.80)$ & 0.060 \\
\hline Length of stay (day) & $1.19 \pm 0.45$ & $1.19 \pm 0.44$ & $1.50 \pm 0.67$ & $1.29 \pm 0.68$ & $0.001 *$ \\
\hline 0 & $103(83.74)$ & 55 (82.09) & $13(59.08)$ & I7I (80.70) & \\
\hline $1-7$ & $17(13.82)$ & II (16.42) & $7(31.82)$ & $35(16.50)$ & \\
\hline$\geq 8$ & $3(2.44)$ & I (I.49) & $2(9.10)$ & $6(2.80)$ & \\
\hline Ambulatory visit (visit) & $6.55 \pm 2.74$ & $6.22 \pm 2.21$ & $6.32 \pm 1.96$ & $6.42 \pm 2.50$ & 0.674 \\
\hline $1-4$ & $15(12.20)$ & II (16.40) & $3(13.60)$ & $29(13.70)$ & \\
\hline $5-7$ & $82(66.70)$ & $44(65.70)$ & $13(59.10)$ & $139(65.60)$ & \\
\hline$\geq 8$ & $26(21.10)$ & $12(17.90)$ & $6(27.30)$ & $44(20.80)$ & \\
\hline BMI $\left(\mathrm{kg} / \mathrm{m}^{2}\right)$ & $23.94 \pm 3.85$ & $23.77 \pm 4.92$ & $23.93 \pm 3.55$ & $23.89 \pm 4.17$ & 0.962 \\
\hline $\mathrm{SBP}(\mathrm{mmHg})$ & $140.38 \pm 14.42$ & $140.54 \pm 13.67$ & $|4| .59 \pm|5.5|$ & $140.55 \pm 14.24$ & 0.935 \\
\hline FBG $(\mathrm{mg} / \mathrm{dL})$ & $103.41 \pm 28.63$ & $109.10 \pm 41.98$ & $|2| .|1| \pm 25.60$ & $107.04 \pm 33.47$ & 0.060 \\
\hline HbAlc (\%), n=74 & $6.46 \pm 0.84$ & $7.10 \pm 1.70$ & $6.80 \pm 1.30$ & $6.72 \pm 1.26$ & 0.151 \\
\hline$<7 \%$ & $29(78.40)$ & $13(56.50)$ & $7(50.00)$ & $49(66.20)$ & \\
\hline$\geq 7 \%$ & $8(21.60)$ & $10(43.50)$ & $7(50.00)$ & $25(33.80)$ & \\
\hline TG (mg/dL) & $159.82 \pm 83.65$ & $165.69 \pm 119.90$ & $180.34 \pm 79.79$ & $163.81 \pm 96.00$ & 0.643 \\
\hline LDL (mg/dL) & || $4.80 \pm 3|.3|$ & $110.48 \pm 39.13$ & $120.89 \pm 39.76$ & $114.07 \pm 34.82$ & 0.449 \\
\hline $\mathrm{HDL}(\mathrm{mg} / \mathrm{dL})$ & $45.82 \pm 11.76$ & $43.80 \pm 8.68$ & $42.18 \pm 10.58$ & $44.80 \pm 10.78$ & 0.227 \\
\hline BUN (mg/dL) & $16.65 \pm 4.97$ & $22.28 \pm 12.54$ & $29.12 \pm 7.77$ & $19.72 \pm 9.29$ & $<0.001 *$ \\
\hline $\operatorname{Scr}(\mathrm{mg} / \mathrm{dL})$ & $1.20 \pm 0.17$ & $1.45 \pm 0.21$ & $2.29 \pm 0.56$ & $1.39 \pm 0.41$ & $<0.001 *$ \\
\hline eGFR $\left(\mathrm{mL} / \mathrm{min} / \mathrm{I} .73 \mathrm{~m}^{2}\right)$ & $53.11 \pm 3.41$ & $39.43 \pm 4.02$ & $25.08 \pm 6.33$ & $45.88 \pm 10.23$ & $<0.001 *$ \\
\hline
\end{tabular}

Note: $*$-value $<0.05$.

Abbreviations: BMI, body mass index; BUN, blood urea nitrogen; CHF, chronic heart failure; CKD, chronic kidney disease; eGFR, estimated glomerular filtration rate; FBG, fasting blood glucose; HbAlc, hemoglobin Al c; HDL, high-density lipoprotein cholesterol; LDL, low-density lipoprotein cholesterol; MI, myocardial infarction; SBP, systolic blood pressure; Scr, serum creatinine; TG, triglyceride; CKD G3a, chronic kidney disease stage G 3a (GFR 45-59 mL/min/l.73 m²); CKD stage 3b, chronic kidney disease stage G 3b (GFR 30-44 mL/min/l.73 $\left.\mathrm{m}^{2}\right)$; CKD G4, chronic kidney disease stage G 4 (GFR I5-29 $\mathrm{mL} / \mathrm{min} / 1.73 \mathrm{~m}^{2}$ ).

more frequently in district hospitals. We found that treatment costs for patients with uncontrolled FBG were substantially higher than for those who met the recommended FBG target of $<130 \mathrm{mg} / \mathrm{dL}^{21}$

About half of the patients had dyslipidemia as a comorbidity. Patients with dyslipidemia had slightly higher treatment costs than those without dyslipidemia. The difference in treatment costs was mainly from drug costs instead of laboratory monitoring, frequency of ambulatory visits, length of hospitalization, or CKD stage.

Again, drug costs were the major component that affected the treatment costs for patients with serum creatinine $\geq 2 \mathrm{mg} /$ dL compared with those with serum creatinine less than 2 $\mathrm{mg} / \mathrm{dL}$. All patients with serum creatinine $\geq 2 \mathrm{mg} / \mathrm{dL}, 92.31 \%$ were categorized into CKD stage G4.

Ambulatory costs for patients aged less than 65 years were higher than those of patients aged more than 65 years. This finding was in line with results of the study conducted in Italy by Turchetti and colleagues. ${ }^{22}$ They reported that CKD patients aged more than 74 years had lower direct medical cost compared to those who aged less than 74 years due to lower costs of diagnostic exams, laboratory tests and hospital cares. ${ }^{22}$

A previous cost-effectiveness study of MDC in CKD patients in Thailand reported direct medical costs of 6265.86 THB per year $^{22}$ (presented in the year 2019), 


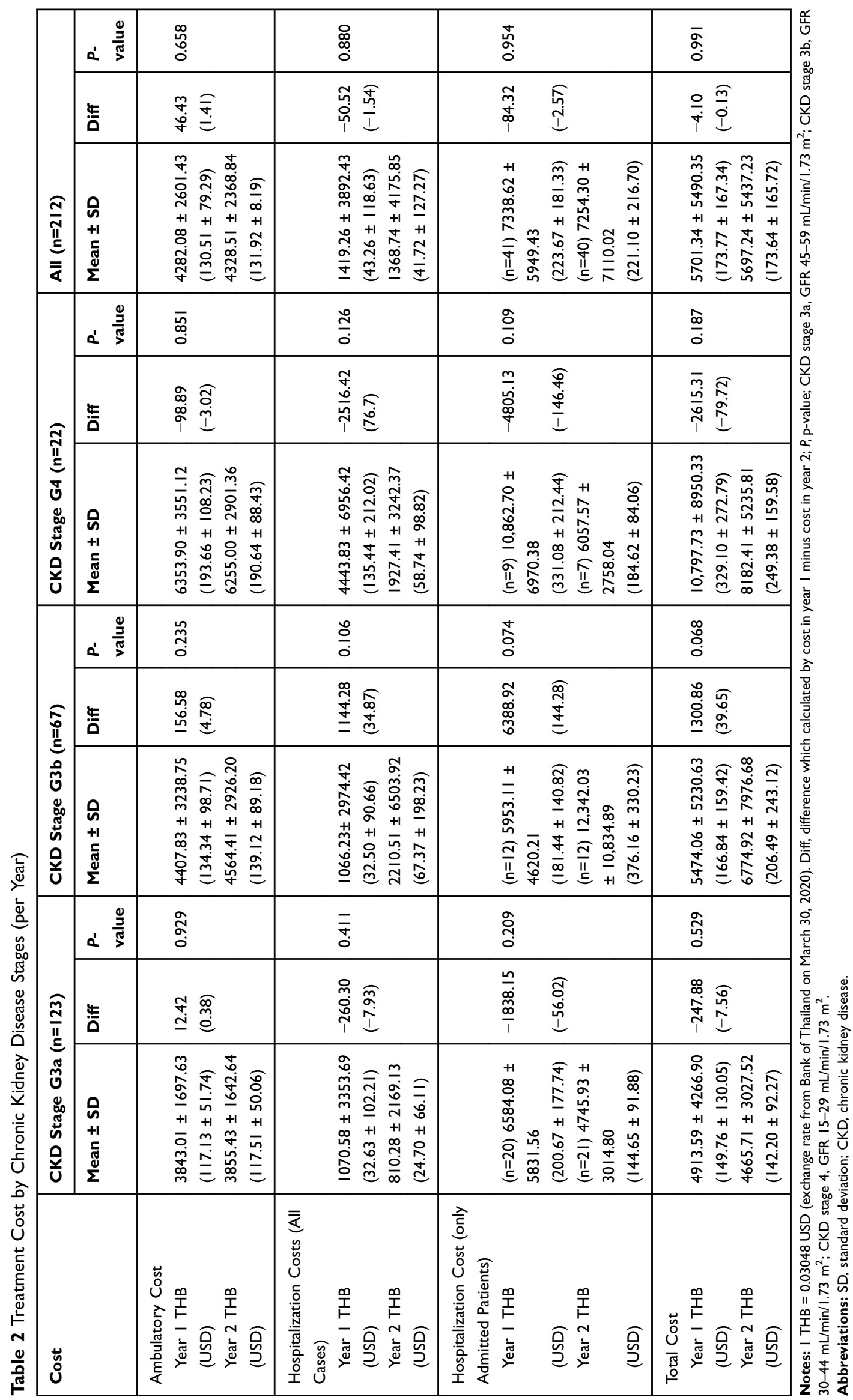




\begin{tabular}{|c|c|c|c|c|c|c|c|c|c|c|c|}
\hline & 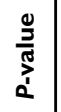 & 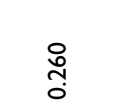 & $\begin{array}{ll}* & \frac{*}{0} \\
& 0 \\
& 0 \\
& 0 \\
v\end{array}$ & 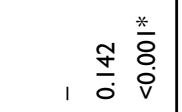 & 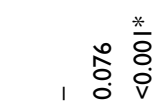 & $\begin{array}{l}\frac{*}{0} \\
\overline{0} \\
\mathrm{v}\end{array}$ & ڤ̂n & $\stackrel{\substack{0 \\
\\
0}}{0}$ & 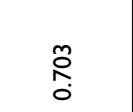 & 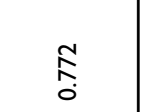 & 总 \\
\hline & 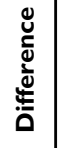 & $\begin{array}{l}\frac{R}{h} \\
\frac{h}{a}\end{array}$ & 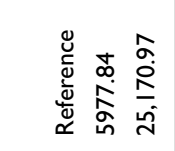 & 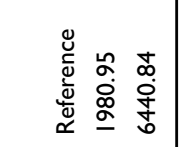 & 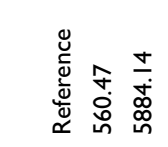 & $\begin{array}{l}\text { N} \\
\stackrel{\rho}{\sigma} \\
\frac{\sigma}{\sigma}\end{array}$ & 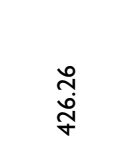 & $\begin{array}{l}\bar{M} \\
\text { bे } \\
\text { b् }\end{array}$ & $\frac{\bar{\infty}}{\bar{p}}$ & 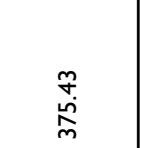 & $\underset{\mathfrak{J}}{\mathfrak{J}}$ \\
\hline 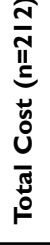 & $\begin{array}{l}0 \\
0 \\
+1 \\
\bar{e} \\
\bar{\Sigma} \\
\Sigma \\
\end{array}$ & 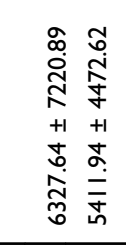 & 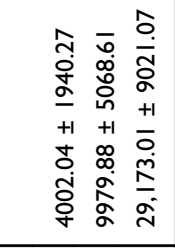 & 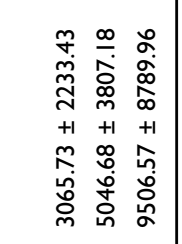 & 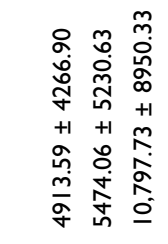 & 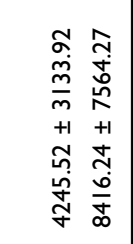 & 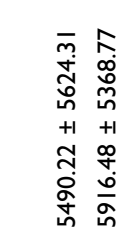 & 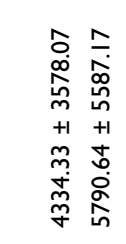 & 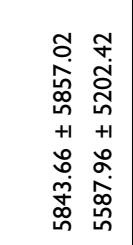 & 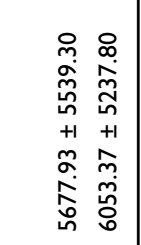 & 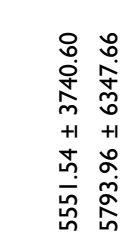 \\
\hline & 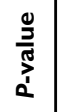 & $\frac{\bar{\infty}}{0}$ & $\frac{\tilde{\infty}}{0}$ & 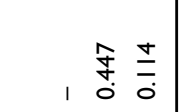 & $\begin{array}{l}\hat{\circ} \\
\hat{0} \\
0 \\
0\end{array}$ & 童 & 疋 & $\frac{7}{0}$ & $\underset{\substack{\text { N } \\
0}}{0}$ & 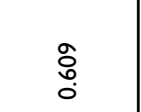 & $\frac{\bar{n}}{0}$ \\
\hline$\widehat{\overline{\mathrm{I}}}$ & 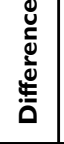 & 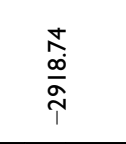 & 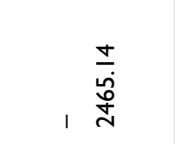 & 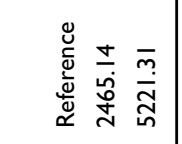 & 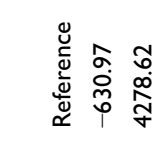 & 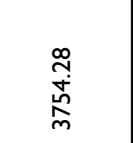 & $\begin{array}{l}0 \\
\text { ơ } \\
\text { مُ }\end{array}$ & 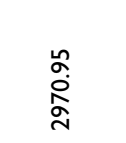 & $\begin{array}{l}\infty \\
\stackrel{\infty}{0} \\
\substack{0 \\
0}\end{array}$ & 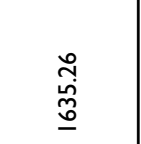 & $\begin{array}{l}\text { òे } \\
\text { م. } \\
\text { N }\end{array}$ \\
\hline 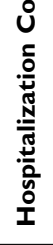 & $\begin{array}{l}\text { Q } \\
+1 \\
+\bar{j} \\
\frac{\delta}{\Sigma}\end{array}$ & 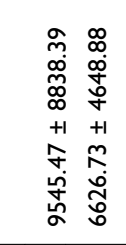 & 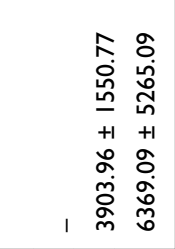 & 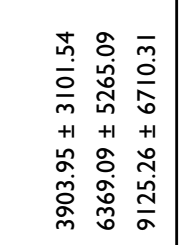 & 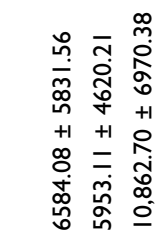 & 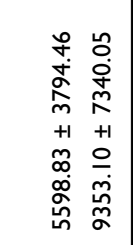 & 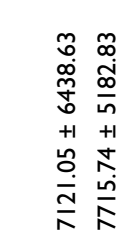 & 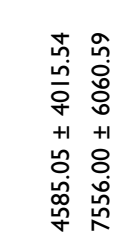 & 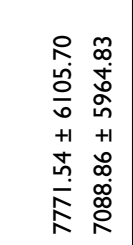 & 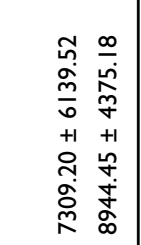 & 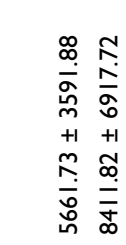 \\
\hline & 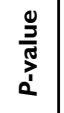 & $\frac{*}{0}$ & , $\begin{array}{l}\stackrel{*}{*} \\
\stackrel{*}{0} \\
0\end{array}$ & 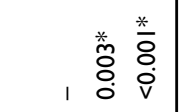 & 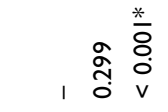 & 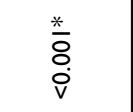 & 壴 & $\frac{\bar{n}}{0}$ & $\stackrel{\infty}{\circ}$ & 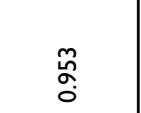 & $\begin{array}{l}\hat{\circ} \\
\text { hn } \\
0\end{array}$ \\
\hline$\widehat{\underline{\Omega}}$ & 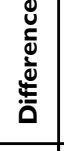 & 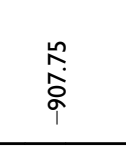 & 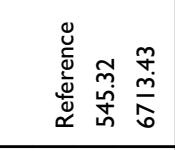 & 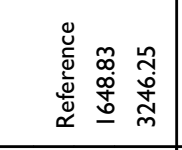 & 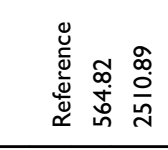 & 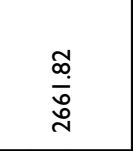 & 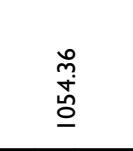 & 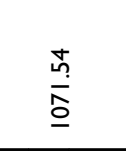 & 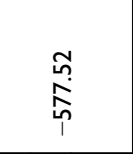 & $\begin{array}{l}\bar{\infty} \\
\stackrel{\infty}{p} \\
\dot{p}\end{array}$ & $\begin{array}{l}\frac{\sigma}{\infty} \\
\substack{0 \\
\rho}\end{array}$ \\
\hline 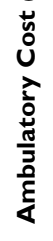 & \begin{tabular}{l|l|}
0 \\
+1 \\
+1 \\
$\bar{\delta}$ \\
$\Sigma$
\end{tabular} & 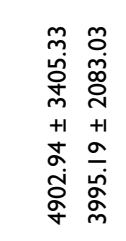 & 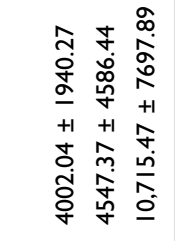 & 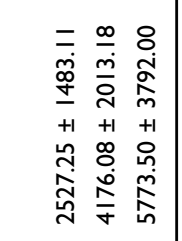 & 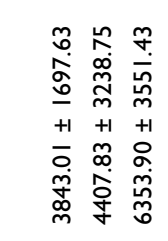 & 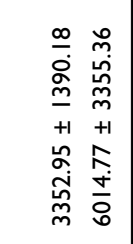 & 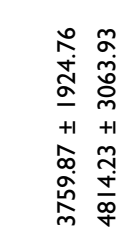 & 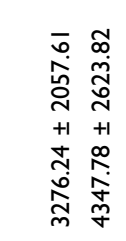 & 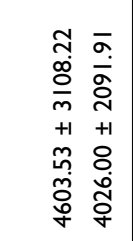 & 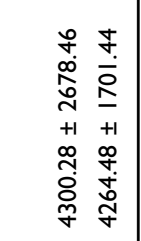 & 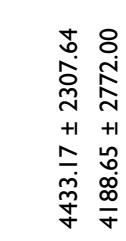 \\
\hline 参 & & 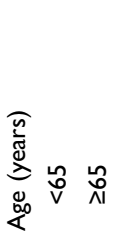 & 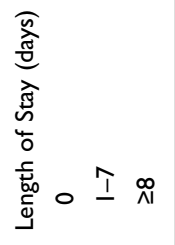 & 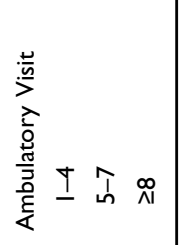 & 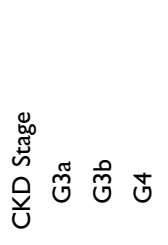 & 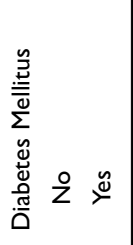 & 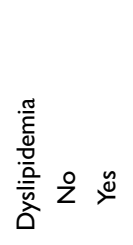 & 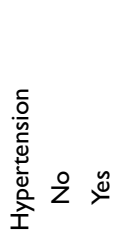 & 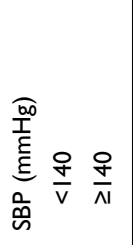 & 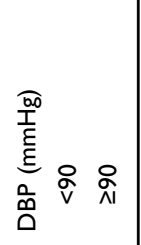 & 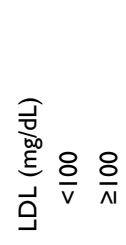 \\
\hline
\end{tabular}




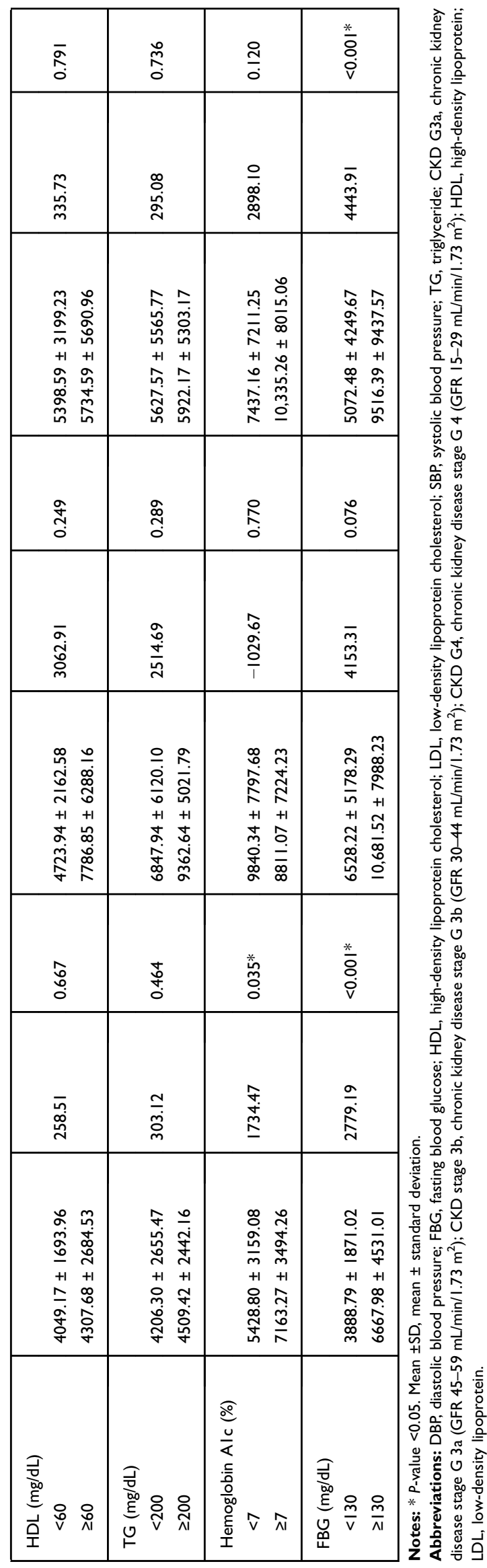

which was slightly greater than our finding (5701.34 THB per year). Srisubat et $\mathrm{al}^{23}$ estimated total costs using unit costs from a district hospital while our study used the accepted national standard unit costs in Thailand. ${ }^{19}$ Various sources of unit costs might lead to unequal results.

Based on our findings, treatment costs do not fluctuate with the implementation of the MDC approach in a district hospital. This might reflect stable clinical status of patients who benefit from MDC. Our results are quite in line with the clinical pieces of evidence from meta-analysis ${ }^{12}$ and other studies ${ }^{6,7,11}$ that report a lower risk of hospitalization and GFR decline.

Approximately $20 \%$ of pre-dialysis CKD patients reached end-stage renal disease (ESRD) within 4-6 years. $^{24,25}$ Most CKD patients also had at least 1 common comorbidity such as diabetes mellitus, dyslipidemia or cardiovascular disease and suffered from many complications. These patients should be referred to receive treatment with the nephrologist.

Some strengths are needed to mention. Even though our center is a community hospital, the MDC team has established for several years due to the readiness of the collaboration among healthcare teams. This leads to a number of CKD patients regularly visiting the MDC clinic each week. In addition, we have the completed data for analyses in this study.

Our study has some limitations. We did not compare the treatment costs of the MDC approach with treatment costs of usual care. This is still a gap for a further experimental study comparing these two approaches, analyzing their benefits in terms of cost and effectiveness. Since this study conducted in a single center in Thailand, the generalizability of our findings to other countries might be limited. However, we believe that in the country that has CKD patient care similar to our study and would like to establish an MDC team, our findings might provide some useful information.

\section{Conclusion}

Total annual treatment costs for 2 years were not different. A more advanced stage of CKD, having diabetes mellitus or dyslipidemia as comorbidities, and uncontrolled fasting blood glucose were significantly associated with increased costs for treatment in patients with pre-dialysis CKD. 
Table 4 Cost Model of Chronic Kidney Disease Treatment

\begin{tabular}{|c|c|c|c|c|c|c|}
\hline & \multicolumn{2}{|c|}{ Unstandardized Coefficients } & \multirow[t]{2}{*}{$\mathbf{t}$} & \multirow[t]{2}{*}{ Sig. } & \multicolumn{2}{|c|}{ 95\% Confidence Interval for B } \\
\hline & B & SE & & & Lower Bound & Upper Bound \\
\hline Constant & -5072.638 & 924.560 & -5.487 & $<0.001$ & -6895.557 & -3249.720 \\
\hline Length of stay & 2191.776 & 65.023 & 33.708 & $<0.001$ & 2063.573 & 2319.980 \\
\hline Ambulatory visit & 456.560 & 50.936 & 8.963 & $<0.001$ & 356.131 & 556.989 \\
\hline Diabetes mellitus & $|290.3| \mid$ & 303.550 & 4.251 & $<0.001$ & 691.813 & 1888.808 \\
\hline Dyslipidemia & 719.157 & 245.677 & 2.927 & 0.004 & 234.765 & 1203.550 \\
\hline Serum creatinine & 1672.233 & 290.772 & 5.751 & $<0.001$ & 1098.930 & 2245.536 \\
\hline Fasting blood glucose & 9.982 & 4.259 & 2.344 & 0.020 & 1.584 & 18.380 \\
\hline Body mass index & 86.737 & 28.777 & 3.014 & 0.003 & 29.998 & | 43.476 \\
\hline
\end{tabular}

Notes: Adjusted $R^{2}=0.906$; probability of $F$-test $<0.00$ I

Abbreviations: SE, standard error; Sig., significance.

\section{Acknowledgment}

The authors would like to thank all staff at Khlong-Lan Hospital for permission to access the hospital database.

\section{Author Contributions}

All authors contributed to data analysis, drafting or revising the article, gave final approval of the version to be published, and agree to be accountable for all aspects of the work.

\section{Disclosure}

The authors declare no conflicts of interest in this work.

\section{References}

1. Hill NR, Fatoba ST, Oke JL, et al. Global prevalence of chronic kidney disease - a systematic review and meta-analysis. PLoS One. 2016;11 (7):e0158765. doi:10.1371/journal.pone.0158765

2. Ingsathit A, Thakkinstian A, Chaiprasert A, et al. Prevalence and risk factors of chronic kidney disease in the Thai adult population: thai SEEK study. Nephrol Dial Transplant. 2010;25(5):1567-1575. doi:10.1093/ndt/gfp669

3. Ku E, Lee BJ, Wei J, Weir MR. Hypertension in CKD: core curriculum 2019. Am J Kidney Dis. 2019;74(1):120-131. doi:10.1053/j. ajkd.2018.12.044

4. Kaewput W, Thongprayoon C, Chewcharat A, et al. Rate of kidney function decline and factors predicting progression of kidney disease in type 2 diabetes mellitus patients with reduced kidney function: a nationwide retrospective cohort study. Ther Apher Dial. 2020. doi:10.1111/1744-9987.13480

5. Manns B, Hemmelgarn B, Tonelli M, et al. The cost of care for people with chronic kidney disease. Can $J$ Kidney Health Dis. 2019;6:2054358119835521. doi:10.1177/2054358119835521

6. Chen YR, Yang Y, Wang SC, et al. Multidisciplinary care improves clinical outcome and reduces medical costs for pre-end-stage renal disease in Taiwan. Nephrology (Carlton). 2014;19(11):699-707. doi:10.1111/nep. 12316

7. Lin E, Chertow GM, Yan B, Malcolm E, Goldhaber-Fiebert JD, Taal MW. Cost-effectiveness of multidisciplinary care in mild to moderate chronic kidney disease in the United States: a modeling study. PLoS Med. 2018;15(3):e1002532. doi:10.1371/journal.pmed.1002532
8. Saran R, Robinson B, Abbott KC, et al. US renal data system 2019 annual data report: epidemiology of kidney disease in the United States. Am J Kidney Dis. 2020;75(1S1):A6-A7. doi:10.1053/j. ajkd.2019.09.003

9. Moreno Velasquez I, Tribaldos Causadias M, Valdes R, et al. Endstage renal disease-financial costs and years of life lost in Panama: a cost-analysis study. BMJ Open. 2019;9(5):e027229. doi:10.1136/ bmjopen-2018-027229

10. Eriksson JK, Neovius M, Jacobson SH, Elinder CG, Hylander B. Healthcare costs in chronic kidney disease and renal replacement therapy: a population-based cohort study in Sweden. BMJ Open. 2016;6(10):e012062. doi:10.1136/bmjopen-2016-012062

11. Lei CC, Lee PH, Hsu YC, et al. Educational intervention in CKD retards disease progression and reduces medical costs for patients with stage 5 CKD. Ren Fail. 2013;35(1):9-16. doi:10.3109/ 0886022X.2012.731997

12. Shi Y, Xiong J, Chen Y, et al. The effectiveness of multidisciplinary care models for patients with chronic kidney disease: a systematic review and meta-analysis. Int Urol Nephrol. 2018;50(2):301-312. doi:10.1007/s11255-017-1679-7

13. Sabariego C, Grill E, Brach M, Fritschka E, Mahlmeister J, Stucki G. Incremental cost-effectiveness analysis of a multidisciplinary renal education program for patients with chronic renal disease. Disabil Rehabil. 2010;32(5):392-401. doi:10.3109/09638280903171584

14. Collister D, Pyne L, Cunningham J, et al. Multidisciplinary chronic kidney disease clinic practices: a scoping review. Can J Kidney Health Dis. 2019;6:2054358119882667. doi:10.1177/2054358119882667

15. Jiamjariyaporn $T$, Ingsathit A, Tungsanga $K$, et al. Effectiveness of integrated care on delaying chronic kidney disease progression in rural communities of Thailand (ESCORT study): rationale and design of the study [NCT01978951]. BMC Nephrol. 2014;15:99. doi:10.1186/1471-2369-15-99

16. Jiamjariyapon $\mathrm{T}$, Ingsathit A, Pongpirul $\mathrm{K}$, et al. Effectiveness of integrated care on delaying progression of stage 3-4 chronic kidney disease in rural communities of Thailand (ESCORT study): a cluster randomized controlled trial. BMC Nephrol. 2017;18(1):83. doi:10.1186/s12882-016-0414-4

17. Kasiske BL, Wheeler DC. KDIGO clinical practice guideline for the evaluation and management of chronic kidney disease. Kidney Int Suppl. 2013;3(1):2.

18. Levey AS, Stevens LA, Schmid CH, et al. A new equation to estimate glomerular filtration rate. Ann Intern Med. 2009;150 (9):604-612. doi:10.7326/0003-4819-150-9-200905050-00006

19. Riewpaiboon A. Standard cost lists for health economic evaluation in Thailand. J Med Assoc Thai. 2014;97(Suppl 5):S127-S134. 
20. Consumer Price Index. Ministry of commerce. Available from: http://www. price.moc.go.th/price/cpi. Accessed January 30, 2020.

21. American Diabetes A. 6. Glycemic targets: standards of medical care in diabetes-2020. Diabetes Care. 2020;43(Suppl 1):S66-S76. doi:10.2337/dc20-S006

22. Turchetti G, Bellelli S, Amato M, et al. The social cost of chronic kidney disease in Italy. Eur $J$ Health Econ. 2017;18(7):847-858. doi:10.1007/s10198-016-0830-1

23. Srisubat A, Jiamjariyaporn T, Chanpitakkul M, et al. Cost-effectiveness of integrated care in patients with chronic kidney disease stage 3 and 4 compared with standard care in rural communities. J Depart Med Serv. 2017;42(6):54-63.
24. Provenzano M, Chiodini P, Minutolo R, et al. Reclassification of chronic kidney disease patients for end-stage renal disease risk by proteinuria indexed to estimated glomerular filtration rate: multicentre prospective study in nephrology clinics. Nephrol Dial Transplant. 2020;35(1):138-147. doi:10.1093/ndt/gfy217

25. Borrelli S, Garofalo C, Mallamaci F, et al. Short-term blood pressure variability in nondialysis chronic kidney disease patients: correlates and prognostic role on the progression of renal disease. J Hypertens. 2018;36(12):2398-2405. doi:10.1097/HJH.0000000000001825

\section{Publish your work in this journal}

ClinicoEconomics and Outcomes Research is an international, peerreviewed open-access journal focusing on Health Technology Assessment, Pharmacoeconomics and Outcomes Research in the areas of diagnosis, medical devices, and clinical, surgical and pharmacologica intervention. The economic impact of health policy and health systems organization also constitute important areas of coverage. The manuscript management system is completely online and includes a very quick and fair peer-review system, which is all easy to use. Visit http://www.dovepress.com/testimonials.php to read real quotes from published authors. 